\title{
The Bost-Connes phase transition and unitary representations
}

\author{
Tyrone Crisp*
}

\begin{abstract}
We construct a family of unitary representations of the $a x+b$ group $\mathbb{Q} \rtimes \mathbb{Q}_{+}^{\times}$. We show that this family of representations exhibits a "phase transition" analogous to that observed by Bost and Connes [2] and then explain how these representations are related to the equilibrium states of Bost and Connes.
\end{abstract}

Mathematics Subject Classification (2010). 22D10, 46L55.

Keywords. Unitary representation, phase transition, Bost-Connes system.

\section{Introduction}

In their paper [2], Bost and Connes constructed a $\mathrm{C}^{*}$-dynamical system from numbertheoretic data, and showed that this system possesses a unique equilibrium state at high temperatures but many distinct equilibria at low temperatures. Since the publication of [2], a number of other systems have been constructed, exhibiting a similar "phase transition": see the recent book of Connes and Marcolli [4] for a discussion of some of the developments and references to others.

The purpose of this note is to describe a manifestation of the Bost-Connes phase transition in the context of unitary representations. We construct a natural family of unitary representations of the rational $a x+b$ group, parametrised by the half-plane $\operatorname{Re} z \geq 0$. We show that this family of representations exhibits a phase transition, in the following sense:

- The representations corresponding to $\operatorname{Re} z=0$ are one-dimensional and mutually inequivalent.

- The representations corresponding to $0<\operatorname{Re} z \leq 1$ are mutually inequivalent, irreducible and infinite-dimensional.

- The representations corresponding to $\operatorname{Re} z>1$ are all mutually equivalent and reducible, with irreducible constituents parametrised by the Galois group $\operatorname{Gal}\left(\mathbb{Q}^{\mathrm{ab}}, \mathbb{Q}\right)$.

\footnotetext{
*Partially supported by NSF DMS 0607879.
} 
The real part of our parameter $z$ thus corresponds to the inverse temperature $\beta$ of Bost and Connes. The imaginary part $\operatorname{Im} z$ is related to the time evolution on the Bost-Connes algebra, as will be explained later in the paper.

The $a x+b$ group that we consider is isomorphic to the semidirect product, $\mathbb{Q} \rtimes \mathbb{Q}_{+}^{\times}$, for the action of the positive rationals on $\mathbb{Q}$ by multiplication. Recall the following standard procedure for producing irreducible representations of such a group: one takes a character $\varphi \in \widehat{\mathbb{Q}}$ and a character $\chi$ of the stabiliser $\left(\mathbb{Q}_{+}^{\times}\right)_{\varphi}$, and induces the representation $\varphi \rtimes \chi$ from $\mathbb{Q} \rtimes\left(\mathbb{Q}_{+}^{\times}\right)_{\varphi}$ to $\mathbb{Q} \rtimes \mathbb{Q}_{+}^{\times}[9], \S 14$. There are two extreme cases of this construction: if $\varphi$ is the trivial character, the representations obtained in this way are one-dimensional; at the other extreme, where the stabiliser of $\varphi$ is trivial, we get $\operatorname{Ind}_{\mathbb{Q}}^{\mathbb{Q} \rtimes \mathbb{Q}_{+}^{\times}} \varphi$. The representations constructed in this paper are, for $\operatorname{Re} z=0$, of the former type, while the irreducible constituents for $\operatorname{Re} z>1$ are of the latter type. The semidirect product $\mathbb{Q} \rtimes \mathbb{Q}_{+}^{\times}$is not regular (in the sense of [9]), so it also possesses irreducible representations not accessible through the above construction; the representations we construct for $0<\operatorname{Re} z \leq 1$ are of this more exotic type.

After establishing some notation, we define in Section 2 the representations in question, and give a precise statement of our main result. Our construction is related, in the case $z=1$, to papers of Blackadar [1] and Matthews [10]. Section 3 contains the proof of our result, part of which relies on an argument due to Neshveyev [11]. In Section 4 we show how our construction connects, via results of Laca [7], [8], to the equilibrium states of Bost and Connes.

This research was carried out as part of the author's doctoral studies at the Pennsylvania State University, under the direction of Nigel Higson. I thank Prof. Higson for his support and encouragement.

Notation. Our notation agrees, for the most part, with that of [2]. We refer to [5], [13] and [3] for more details about adeles and ideles. $\mathcal{A}$ and $\mathcal{A}^{\times}$denote, respectively, the locally compact ring of finite adeles of $\mathbb{Q}$, and the locally compact group of finite ideles. Recall the definition of $\mathcal{A}$ and $\mathcal{A}^{\times}$as restricted direct products over the primes,

$$
\mathcal{A}=\prod_{p}^{\prime}\left(\mathbb{Q}_{p}, \mathbb{Z}_{p}\right), \quad \mathcal{A}^{\times}=\prod_{p}^{\prime}\left(\mathbb{Q}_{p}^{\times}, \mathbb{Z}_{p}^{\times}\right) .
$$

We normalise additive Haar measure on each $\mathbb{Q}_{p}$ so that $\mathbb{Z}_{p}$ has measure one; the product of these measures is a Haar measure on the additive group $\mathcal{A}$, giving measure one to the compact open subring $\mathcal{R}=\prod_{p} \mathbb{Z}_{p}$. Similarly, we normalise Haar measure on $\mathbb{Q}_{p}^{\times}$so that $\mathbb{Z}_{p}^{\times}$has measure one; the product measure on $\mathcal{A}^{\times}$is a Haar measure, giving measure one to the compact open subgroup $W=\prod_{p} \mathbb{Z}_{p}^{\times}$.

$\mathbb{Q}$ embeds diagonally into $\mathcal{A}$ as a dense subring, and $\mathbb{Q}_{+}^{\times}$embeds diagonally into $\mathcal{A}^{\times}$as a discrete and co-compact subgroup. There is an "absolute value" $|\cdot|: \mathscr{A}^{\times} \rightarrow$ $\mathbb{Q}_{+}^{\times}$, which may be defined as the Radon-Nikodym derivative

$$
|a|=\frac{\mathrm{d}(a x)}{\mathrm{d}(x)},
$$


where $\mathrm{d} x$ is the additive Haar measure on $\mathcal{A}$. Alternatively, $|\cdot|$ may be defined as the product of the $p$-adic absolute values. One has $|a|=a^{-1}$ for all $a \in \mathbb{Q}_{+}^{\times}$, and $W$ is the kernel of the absolute value. In this way, we can decompose $\mathcal{A}^{\times}$as the direct product $\mathbb{Q}_{+}^{\times} \times W$. Note that $W \cong \operatorname{Gal}\left(\mathbb{Q}^{\mathrm{ab}}, \mathbb{Q}\right)$ (by the Kronecker-Weber theorem, [3], VII, §5.7).

The Schwartz-Bruhat space $S(\mathcal{A})$ can be defined as the space of locally constant, compactly supported, complex-valued functions on $\mathcal{A}$. It is a $*$-algebra, under convolution (with respect to additive Haar measure) and the involution $f^{*}(x)=\overline{f(-x)}$. $\delta(\mathcal{A})$ is a direct limit of finite-dimensional spaces, so it is nuclear in the direct-limit topology, and every linear functional is continuous.

The additive group $\mathcal{A}$ is (non-canonically) self-dual. We fix an identification $\mathcal{A} \cong \hat{\mathcal{A}}$ as follows. For each prime $p$, let $\psi_{p}$ be a character on $\mathbb{Q}_{p}$ that is trivial on $\mathbb{Z}_{p}$ but non-trivial on $p^{-1} \mathbb{Z}_{p}$. Then $\psi:=\bigotimes_{p} \psi_{p}$ is a character of $\mathcal{A}$, and we associate to each $a \in \mathcal{A}$ the character $\psi_{a}: x \mapsto \psi(a x)$. The Fourier transform arising from this identification, $\hat{f}(x)=\int_{\mathscr{A}} f(y) \psi(x y) \mathrm{d} y$, is a linear automorphism of $S(\mathcal{A})$, and our character $\psi$ was chosen so that this automorphism fixes the characteristic function $f_{\mathcal{R}}$ of $\mathcal{R}$.

\section{The phase transition}

We will construct representations of the " $a x+b$ group"

$$
P_{\mathbb{Q}}^{+}=\left\{\left(\begin{array}{ll}
1 & b \\
0 & a
\end{array}\right) \mid a \in \mathbb{Q}_{+}^{\times}, b \in \mathbb{Q}\right\} .
$$

This group embeds into the adelic $a x+b$ group,

$$
P_{\mathcal{A}}=\left\{\left(\begin{array}{ll}
1 & b \\
0 & a
\end{array}\right) \mid a \in \mathcal{A}^{\times}, b \in \mathcal{A}\right\} .
$$

$P_{\mathcal{A}}$ has a natural action on $\mathcal{A}$, by affine transformations, giving rise to a linear representation on $S(\mathcal{A})$,

$$
\left(\begin{array}{ll}
1 & b \\
0 & a
\end{array}\right) f(x)=f(a x+b)
$$

The group $P_{\mathcal{A}}$ is not unimodular: its modular function is $\left(\begin{array}{ll}1 & b \\ 0 & a\end{array}\right) \mapsto|a|$. Twisting the above representation by the complex powers of this function, we obtain a family of representations $\alpha_{z}$ parametrised by $z \in \mathbb{C}$. In order to make our parametrisation agree with that of [2], we define

$$
\alpha_{z}\left(\begin{array}{ll}
1 & b \\
0 & a
\end{array}\right) f(x)=|a|^{1-\frac{z}{2}} f(a x+b) .
$$


This is a standard construction in representation theory: whenever a group $G$ acts on a space $X$ admitting a quasi-invariant measure, one obtains a family of (not necessarily unitary) representations of $G$ on an appropriate space of functions on $X$, by twisting the usual representation by the complex powers of the Radon-Nikodym derivative. See, for example, the construction of the principal and complementary series for $\mathrm{SL}_{2}$ in [5], Chapter 2, §3.

We first describe which of the representations $\alpha_{z}$ are unitarisable, in the sense that $S(\mathcal{A})$ admits a non-trivial (but possibly degenerate), positive, $\alpha_{z}$-invariant hermitian form.

Lemma 2.1. If $\operatorname{Re} z<0, \alpha_{z}$ is not unitarisable.

If $\operatorname{Re} z \geq 0$, then $\alpha_{z}$ is unitarisable, and any two unitarisations of $\alpha_{z}$ are equivalent. If $\operatorname{Re} z=0$, the corresponding unitary representation is one-dimensional. If $\operatorname{Re} z>0$, the representation is infinite-dimensional.

The proof is given in the next section.

We will focus on the unitary case, $\operatorname{Re} z \geq 0$, and for the rest of this section $\alpha_{z}$ denotes the unitary representation of $P_{\mathcal{A}}$ given by Lemma 2.1. We consider the unitary representations

$$
\operatorname{Res} \alpha_{z}=\operatorname{Res}_{P_{\mathbb{Q}}^{+}}^{P_{\mathcal{A}}} \alpha_{z}
$$

of $P_{\mathbb{Q}}^{+}$, obtained by restricting $\alpha_{z}$ to this subgroup.

Theorem 2.2. If $\operatorname{Re} z=0$, then $\operatorname{Res} \alpha_{z}$ is equivalent to the one-dimensional representation $\left(\begin{array}{ll}1 & b \\ 0 & a\end{array}\right) \mapsto a^{i \operatorname{Im} z / 2}$.

If $0<\operatorname{Re} z \leq 1$, then $\operatorname{Res} \alpha_{z}$ is irreducible and infinite-dimensional, and is not induced by any character of $\mathbb{Q}$ (as described in Section 1 ). If $z \neq z^{\prime}$ in this strip, then $\operatorname{Res} \alpha_{z}$ and $\operatorname{Res} \alpha_{z^{\prime}}$ are inequivalent.

If $\operatorname{Re} z>1$, then there is a direct-integral decomposition

$$
\operatorname{Res} \alpha_{z} \cong \int_{W}^{\oplus} \operatorname{Ind}_{\mathbb{Q}}^{P_{\mathbb{Q}}^{+}} \psi_{w} \mathrm{~d} w,
$$

and the representations Ind $\psi_{w}$ are mutually inequivalent and irreducible. In particular, $\operatorname{Res} \alpha_{z}$ and $\operatorname{Res} \alpha_{z^{\prime}}$ are unitarily equivalent whenever $\operatorname{Re} z, \operatorname{Re} z^{\prime}>1$.

Remarks 2.3. (1) In the course of proving Theorem 2.2, we will see that the representations $\alpha_{z}$ themselves undergo a "phase transition" of a similar (albeit less drastic) kind: $\alpha_{z}$ is irreducible whenever $\operatorname{Re} z \geq 0$; for $\operatorname{Re} z=0, \alpha_{z}$ is one-dimensional; for $0<\operatorname{Re} z \leq 1, \alpha_{z}$ is irreducible, and is not induced by any character of $\mathcal{A}$; for $\operatorname{Re} z>1, \alpha_{z} \cong \operatorname{Ind}_{\mathcal{A}}^{P_{\mathcal{A}}} \psi$.

(2) $P_{\mathbb{Q}}^{+}$is not closed in $P_{\mathcal{A}}$; its closure is the subgroup

$$
\mathcal{A} \rtimes \mathbb{Q}_{+}^{\times}=\left\{\left(\begin{array}{ll}
1 & b \\
0 & a
\end{array}\right) \mid a \in \mathbb{Q}_{+}^{\times}, b \in \mathcal{A}\right\},
$$


and the decomposition $\mathcal{A}^{\times} \cong \mathbb{Q}_{+}^{\times} \times W$ gives

$$
P_{\mathcal{A}} \cong\left(\mathcal{A} \rtimes \mathbb{Q}_{+}^{\times}\right) \rtimes W .
$$

From a representation-theoretic point of view, it might appear more natural to consider the restriction of $\alpha_{z}$ to the closed subgroup $\mathcal{A} \rtimes \mathbb{Q}_{+}^{\times}$. The statement of Theorem 2.2 would remain the same - the representations $\alpha_{z}$ are continuous, so questions about their reducibility and equivalence have the same answers for $\mathcal{A} \rtimes \mathbb{Q}_{+}^{\times}$as for its dense subgroup $P_{\mathbb{Q}}^{+}$.

\section{Proofs}

Proof of Lemma 2.1. Fix $z$. Every translation-invariant hermitian form on $S(\mathcal{A})$ has the form

$$
\left\langle f_{1}, f_{2}\right\rangle=D\left(f_{1} * f_{2}^{*}\right)
$$

for some tempered distribution $D \in S(\mathcal{A})^{*}$ (this follows from the kernel theorem, as in [6], II, §3.5). If such a form is to be invariant also under the group $\alpha_{z}\left(\begin{array}{cc}1 & 0 \\ 0 & A^{\times}\end{array}\right)$, then $D$ must satisfy the homogeneity equation

$$
D(f(a x))=|a|^{\operatorname{Re} z-1} D(f(x)) \quad \text { for all } a \in \mathcal{A}^{\times}, f \in \mathcal{S}(\mathcal{A}) .
$$

Weil showed in [15] that the space of distributions satisfying this equation is onedimensional. Moreover, the assertions about positivity and degeneracy follow from the explicit construction of these distributions described in [15]. In particular, for $\operatorname{Re} z=0$ the distribution in question is additive Haar measure, or in other words the Fourier transform of the Dirac distribution at 0 , and so the resulting inner product is degenerate on a subspace of codimension 1 .

The assertion of Theorem 2.2 in the case $\operatorname{Re} z=0$ is now easily dealt with: the map $\delta(\mathcal{A}) \rightarrow \mathbb{C}$ given by integration with respect to Haar measure establishes a unitary equivalence between $\operatorname{Res} \alpha_{z}$ and the given one-dimensional representation.

For each $z$ with $\operatorname{Re} z>0$, let us fix a choice of $\alpha_{z}$-invariant inner product on $\delta(\mathcal{A})$ by requiring that the characteristic function $f_{\mathcal{R}}$ of $\mathcal{R}$ be a unit vector. From now on, $\alpha_{z}$ will denote the corresponding unitary representation.

We turn to the proof of Theorem 2.2. Rather than work with the representations $\alpha_{z}$ directly, it will be convenient to consider a family of unitarily equivalent representations, which we now define.

For each $\beta>0$, let $\mu_{\beta}$ be the regular Borel measure on $\mathcal{A}$ characterised by the properties

(1) $\mathrm{d} \mu_{\beta}(a x)=|a|^{\beta} \mathrm{d} \mu_{\beta}(x)$ for every $a \in \mathcal{A}^{\times}$,

(2) $\mu_{\beta}(\mathcal{R})=1$. 
(Note that each $\mu_{\beta}$ is the Fourier transform of a distribution satisfying (3.1) with $\beta=\operatorname{Re} z$. The existence and uniqueness of such a measure thus follows from [15].)

For each $z \in \mathbb{C}$ with $\operatorname{Re} z>0$, define a unitary representation $\hat{\alpha}_{z}$ of $P_{\mathcal{A}}$ on $L^{2}\left(\mathcal{A}, \mu_{\operatorname{Re} z}\right)$ by

$$
\hat{\alpha}_{z}\left(\begin{array}{ll}
1 & b \\
0 & a
\end{array}\right) f(x)=|a|^{-z / 2} \bar{\psi}\left(a^{-1} x b\right) f\left(a^{-1} x\right) .
$$

A routine calculation shows that, under our choice of normalisations, the Fourier transform $S(\mathcal{A}) \rightarrow S(\mathcal{A})$ implements a unitary equivalence between $\alpha_{z}$ and $\hat{\alpha}_{z}$.

Theorem 2.2 will be proved as a consequence of the following lemma, which is a generalisation of [11], Proposition, with a similar proof (cf. also [2], §7).

Lemma 3.2. Suppose that $0<\beta \leq 1$. For each $w \in \mathbb{C}$ with $\operatorname{Re} w \geq 0$, let $H_{\beta, w}$ denote the subspace of $L^{2}\left(\mathcal{R}, \mu_{\beta}\right)$ consisting of functions $f$ with $f(a x)=a^{-w} f(x)$ for all positive integers $a$. Then

$$
\operatorname{dim} H_{\beta, w}= \begin{cases}1 & \text { if } w=0 \\ 0 & \text { otherwise }\end{cases}
$$

Proof. The case $w=0$ was shown by Neshveyev in [11]. The same proof applies, with mostly cosmetic changes, to the case $w \neq 0$. Instead of reproducing Neshveyev's argument here, let us point out only that part which is responsible for the difference between the two cases.

Following Neshveyev, one finds that $H_{\beta, w}=0$ if and only if

$$
\lim _{t \rightarrow 1^{+}} \frac{L(\chi, t+\bar{w})}{\zeta(t)}=0
$$

for all Dirichlet characters $\chi$, where $L$ is the corresponding Dirichlet $L$-function and $\zeta$ is Riemann's function. Elementary properties of Dirichlet series (as explained in [12], VI, $\$ 3$, for instance) imply that if $w \neq 0$, the numerator in the limit remains bounded, while the denominator diverges. So the limit is equal to zero for all $\chi$ when $w \neq 0$.

(Note that the same conclusion is not valid when $w=0$ and $\chi$ is a principal character: in this case both the numerator and the denominator have a simple pole at $t=1$.)

Proof of Theorem 2.2. We already took care of the case of $\operatorname{Re} z=0$ above.

Suppose $0<\operatorname{Re} z \leq 1$. Any bounded operator on $L^{2}\left(\mathcal{A}, \mu_{\operatorname{Re} z}\right)$ commuting with $\hat{\alpha}_{z}\left(\begin{array}{cc}1 & \mathbb{Q} \\ 0 & 1\end{array}\right)$ is given by pointwise multiplication by some function $f \in L^{\infty}\left(\mathcal{A}, \mu_{\operatorname{Re} z}\right)$ (because $\mathbb{Q}$ is dense in $\mathcal{A}$ ). If such an operator is to commute with $\hat{\alpha}_{z}\left(\begin{array}{ll}1 & 0 \\ 0 & \mathbb{Q}_{+}^{\times}\end{array}\right)$, we must have $f(a x)=f(x)$ for all $a \in \mathbb{Q}_{+}^{\times}$. Any such function is determined by its 
restriction to $\mathcal{R}$, and Lemma 3.2 then implies that $f$ is a constant, and so $\operatorname{Res} \alpha_{z}$ is irreducible.

Similarly, if $t \in \mathbb{R}$, then any intertwining operator from $\operatorname{Res} \alpha_{z}$ to $\operatorname{Res} \alpha_{z+i t}$ is given by an element of $H_{\operatorname{Re} z,-i t / 2}$, so Lemma 3.2 implies that these two representations are inequivalent unless $t=0$.

Lemma 3.2 also implies that for $0<\beta<\beta^{\prime} \leq 1$, the measures $\mu_{\beta}$ and $\mu_{\beta^{\prime}}$ are inequivalent. Indeed, if they were equivalent then we would have

$$
\left.\frac{\mathrm{d} \mu_{\beta^{\prime}}}{\mathrm{d} \mu_{\beta}}\right|_{\mathcal{R}} \in H_{\beta, \beta^{\prime}-\beta}=0 .
$$

If $z, z^{\prime}$ have $0<\operatorname{Re} z<\operatorname{Re} z^{\prime} \leq 1$, it follows that $\operatorname{Res} \alpha_{z}$ and $\operatorname{Res} \alpha_{z^{\prime}}$ are inequivalent (they are even inequivalent as representations of $\mathbb{Q}$ ). To finish with the case $0<\operatorname{Re} z \leq 1$, we note that the representation $\operatorname{Res} \alpha_{z}$ cannot be obtained by the "standard procedure" described in Section 1 because its restriction to $\mathbb{Q}$ corresponds to a properly ergodic measure class on $\widehat{\mathbb{Q}}$.

Now suppose that $\operatorname{Re} z>1$. In this case the measure $\mu_{\operatorname{Re} z}$ is concentrated on the subset $\mathcal{A}^{\times} \subset \mathcal{A}$ [11], and the operator

$$
I_{z}: L^{2}\left(\mathcal{A}, \mu_{\operatorname{Re} z}\right) \rightarrow L^{2}\left(\mathcal{A}^{\times}\right), \quad I_{z} f(x)=|x|^{z / 2} f(x),
$$

is a positive multiple of a unitary. $I_{z}$ intertwines $\hat{\alpha}_{z}$ with the unitary representation $\operatorname{Ind}_{\mathcal{A}}^{P_{\mathcal{A}}} \psi$. Transitivity of induction, coupled with the decomposition

$$
P_{\mathcal{A}} \cong \overline{P_{\mathbb{Q}}^{+}} \rtimes W,
$$

now implies that

$$
\operatorname{Res} \alpha_{z} \cong \int_{W}^{\oplus} \operatorname{Ind}_{\mathbb{Q}}^{P_{\mathbb{Q}}^{+}} \psi_{w} \mathrm{~d} w .
$$

Each of the representations appearing in this direct integral is irreducible, because no element of $\mathbb{Q}_{+}^{\times}$fixes any $\psi_{w}$. They are all mutually inequivalent, because the characters $\psi_{w}$ and $\psi_{w^{\prime}}$ lie in distinct $\mathbb{Q}_{+}^{\times}$-orbits whenever $w \neq w^{\prime}$.

\section{KMS states on the Bost-Connes $\mathrm{C}^{*}$-algebra}

We now briefly explain how the representations appearing in Theorem 2.2 are related, via work of Laca [7], [8], to the results of [2]. The arguments become most transparent when considered from the adelic point of view, and we begin by recalling this perspective on the Bost-Connes algebra and its representations.

Bost and Connes consider the Hecke $\mathrm{C}^{*}$-algebra $C^{*}\left(P_{\mathbb{Q}}^{+}, P_{\mathbb{Z}}^{+}\right)$associated to the almost normal subgroup $P_{\mathbb{Z}}^{+}=\left(\begin{array}{ll}1 & \mathbb{Z} \\ 0 & 1\end{array}\right) \subset P_{\mathbb{Q}}^{+}$. A general result of Tzanev [14], which was observed in this particular case by Laca [8], implies that the Bost-Connes 
$\mathrm{C}^{*}$-algebra is isomorphic to the full corner of the group $\mathrm{C}^{*}$-algebra $C^{*}\left(\mathcal{A} \rtimes \mathbb{Q}_{+}^{\times}\right)$determined by the projection $e_{1} \otimes f_{\mathcal{R}} \in C_{c}\left(\mathbb{Q}_{+}^{\times} \times \mathcal{A}\right)$ (here $e_{1}$ denotes the characteristic function of $1 \in \mathbb{Q}_{+}^{\times}$, and $f_{\mathcal{R}}$ the characteristic function of $\mathcal{R} \subset \mathcal{A}$ ).

Suppose that $\pi$ is a unitary representation of $P_{\mathbb{Q}}^{+}$on a Hilbert space $\mathscr{H}$. If this representation extends to a unitary representation of $\mathcal{A} \rtimes \mathbb{Q}_{+}^{\times}$, then it can be integrated to a $*$-representation of $C^{*}\left(\mathcal{A} \rtimes \mathbb{Q}_{+}^{\times}\right)$, and then compressed by $\pi\left(e_{1} \otimes f_{\mathcal{R}}\right)$ to obtain a $*$-representation of the Hecke algebra $C^{*}\left(P_{\mathbb{Q}}^{+}, P_{\mathbb{Z}}^{+}\right)$on the space $\pi\left(e_{1} \otimes f_{\mathcal{R}}\right) \mathscr{H}=$ $\mathscr{H}^{\mathcal{R}}$ of $\mathcal{R}$-fixed vectors. We use the same symbol to denote both the original unitary representation of $P_{\mathbb{Q}}^{+}$, and the resulting representation of $C^{*}\left(P_{\mathbb{Q}}^{+}, P_{\mathbb{Z}}^{+}\right)$.

Let $\rho$ denote the right-regular representation of $\mathbb{Q}_{+}^{\times}$, which we view as a representation of $P_{\mathbb{Q}}^{+}$via the quotient map $P_{\mathbb{Q}}^{+} \rightarrow \mathbb{Q}_{+}^{\times}$. For each $z \in \mathbb{C}$ with $\operatorname{Re} z>0$, consider the unitary representation $\rho \otimes \operatorname{Res} \alpha_{z}$ of $P_{\mathbb{Q}}^{+}$. These representations all extend to $\mathcal{A} \rtimes \mathbb{Q}_{+}^{\times}$and so induce $*$-representations of the Bost-Connes $C^{*}$-algebra. The function $e_{1} \otimes f_{\mathcal{R}}$ may be viewed as a vector $f_{z}$ in the representation $\rho \otimes \operatorname{Res} \alpha_{z}$, and our choices of normalisation ensure that $f_{z}$ is a unit vector, fixed by the subgroup $\mathcal{R} \subset \mathcal{A} \rtimes \mathbb{Q}_{+}^{\times}$.

Proposition 4.1. For each $z$ with $\operatorname{Re} z>0$, let $\varphi_{z}$ denote the unique $W$-invariant $K M S_{\mathrm{Re} z}$ state on $C^{*}\left(P_{\mathbb{Q}}^{+}, P_{\mathbb{Z}}^{+}\right)$. The GNS representation of $\varphi_{z}$ is equivalent to the representation $\rho \otimes \operatorname{Res} \alpha_{z}$, with $f_{z}$ corresponding to the distinguished cyclic vector. In particular,

$$
\varphi_{z}(a)=\left\langle\rho \otimes \operatorname{Res} \alpha_{z}(a) f_{z}, f_{z}\right\rangle .
$$

Proof. Let $\hat{f}_{z}$ denote the vector $e_{1} \otimes f_{\mathcal{R}}$ in $\ell^{2}\left(\mathbb{Q}_{+}^{\times}\right) \otimes L^{2}\left(\mathcal{A}, \mu_{\operatorname{Re} z}\right)$; the notation is justified by the fact that the equivalence $\alpha_{z} \rightarrow \hat{\alpha}_{z}$ fixes $f_{\mathcal{R}}$.

Let $\pi_{z}$ denote the unitary representation of $\mathcal{A}$ associated with the measure $\mu_{\operatorname{Re} z}$ on $\mathcal{A}=\hat{\mathcal{A}}$ (in other words, $\pi_{z}$ is the restriction from $P_{\mathcal{A}}$ to $\mathcal{A}$ of the representation $\hat{\alpha}_{z}$ ). Results of Laca ([7], Theorem 34, [8], Proposition 3.1) imply that the $W$-invariant $\mathrm{KMS}_{\operatorname{Re} z}$ state on $C^{*}\left(P_{\mathbb{Q}}^{+}, P_{\mathbb{Z}}^{+}\right)$is the vector state in the representation

$$
\operatorname{Ind}_{\mathcal{A}}^{\mathcal{A} \times \mathbb{Q}_{+}^{\times}} \pi_{z}: C^{*}\left(P_{\mathbb{Q}}^{+}, P_{\mathbb{Z}}^{+}\right) \rightarrow \mathscr{B}\left(\ell^{2}\left(\mathbb{Q}_{+}^{\times}\right) \otimes L^{2}\left(\mathcal{A}, \mu_{\operatorname{Re} z}\right)\right)^{\mathcal{R}}
$$

corresponding to the $\mathcal{R}$-fixed cyclic unit vector $\hat{f}_{z}$.

We therefore seek a unitary operator $U$ on $\ell^{2}\left(\mathbb{Q}_{+}^{\times}\right) \otimes L^{2}\left(\mathcal{A}, \mu_{\operatorname{Re} z}\right)$ that intertwines the representations $\operatorname{Ind}_{\mathbb{Q}}^{P_{\mathbb{Q}}^{+}} \pi_{z}$ and $\rho \otimes \operatorname{Res} \hat{\alpha}_{z}$, and fixes the vector $\hat{f}_{z}$. The formula

$$
U f(q, x)=q^{-z / 2} f(q, q x),
$$

for $q \in \mathbb{Q}_{+}^{\times}, x \in \mathcal{A}$ and $f \in C_{c}\left(\mathbb{Q}_{+}^{\times} \times \mathcal{A}\right)$, defines such an operator.

We conclude with some remarks on the interpretation of our parameters $\operatorname{Re} z$ and $\operatorname{Im} z$ in the thermodynamical setting of Bost and Connes. Proposition 4.1 shows how 
our parameter $\operatorname{Re} z$ may be identified with the inverse temperature $\beta$ of [2]. The parameter $\operatorname{Im} z$ corresponds to the time evolution on the Bost-Connes algebra, in the following sense. Denoting by $\left\{\sigma_{t} \mid t \in \mathbb{R}\right\}$ the one-parameter automorphism group of $C^{*}\left(P_{\mathbb{Q}}^{+}, P_{\mathbb{Q}}^{+}\right)$defined in [2], and viewing each $\operatorname{Res} \alpha_{z}$ as a representation of this $\mathrm{C}^{*}$-algebra, we have

$$
\operatorname{Res} \alpha_{z} \circ \sigma_{t}=\operatorname{Res} \alpha_{z+2 i t} .
$$

The assertions in Theorem 2.2 regarding (in)equivalence of the representations $\operatorname{Res} \alpha_{z}$ and $\operatorname{Res} \alpha_{z+i t}$ thus admit the following "dynamical" formulation:

Proposition 4.2. If $\operatorname{Re} z>1$, then the representation $\operatorname{Res} \alpha_{z}$ can be incorporated into a covariant representation $\left(\operatorname{Res} \alpha_{z}, U\right)$ of the $\mathrm{C}^{*}$-dynamical system $\left(C^{*}\left(P_{\mathbb{Q}}^{+}, P_{\mathbb{Z}}^{+}\right), \sigma\right)$. If $0<\operatorname{Re} z \leq 1$, then $\operatorname{Res} \alpha_{z}$ does not admit such a covariant extension.

(By contrast, the representation $\rho \otimes \operatorname{Res} \alpha_{z}$ can always be incorporated into a covariant representation since it is the GNS representation for the $\sigma$-invariant state $\left.\varphi_{z} \cdot\right)$

\section{References}

[1] B. E. Blackadar, The regular representation of restricted direct product groups. J. Functional Analysis 25 (1977), 267-274. Zbl 0364.22004 MR 0439979

[2] J.-B. Bost and A. Connes, Hecke algebras, type III factors and phase transitions with spontaneous symmetry breaking in number theory. Selecta Math. (N.S.) 1 (1995), 411-457. Zbl 0842.46040 MR 1366621

[3] J. W. S. Cassels and A. Fröhlich (Eds.), Algebraic number theory. Academic Press, London 1967. Zbl 0153.07403 MR 0215665

[4] A. Connes and M. Marcolli, Noncommutative geometry, quantum fields and motives. Amer. Math. Soc. Colloq. Publ. 55, Amer. Math. Soc., Providence, RI, 2008. Zbl 1209.58007 MR 2371808

[5] I. M. Gel'fand, M. I. Graev, and I. I. Pyatetskii-Shapiro, Representation theory and automorphic functions. Academic Press, Boston 1990. Zbl 0718.11022 MR 1071179

[6] I. M. Gel'fand and N. Ya. Vilenkin, Applications of harmonic analysis. Academic Press, New York 1964. Zbl 0136.11201 MR 0173945

[7] M. Laca, Semigroups of *-endomorphisms, Dirichlet series, and phase transitions. $J$. Funct. Anal. 152 (1998), 330-378. Zbl 0957.46039 MR 1608003

[8] M. Laca, From endomorphisms to automorphisms and back: dilations and full corners. J. London Math. Soc. (2) 61 (2000), 893-904. Zbl 0973.46066 MR 1766113

[9] G. W. Mackey, Induced representations of locally compact groups I. Ann. of Math. (2) 55 (1952), 101-139. Zbl 0046.11601 MR 0044536

[10] C. R. Matthews, Spectral analysis of the action of ideles on adeles. J. London Math. Soc. (2) 32 (1985), 392-398. Zbl 0614.12009 MR 825913 
[11] S. Neshveyev, Ergodicity of the action of the positive rationals on the group of finite adeles and the Bost-Connes phase transition theorem. Proc. Amer. Math. Soc. 130 (2002), 2999-3003. Zbl 1031.46077 MR 1908923

[12] J.-P. Serre, A course in arithmetic. Graduate Texts in Math. 7, Springer-Verlag, New York 1973. Zbl 0256.12001 MR 0344216

[13] J. Tate, Fourier analysis in number fields and Hecke's zeta function. In Algebraic number theory. Academic Press, London 1967, 305--347. Zbl 0153.07403 MR 0217026

[14] K. Tzanev, Hecke $C^{*}$-algebras and amenability. J. Operator Theory 50 (2003), 169-178. Zbl 1036.46054 MR 2015025

[15] A. Weil, Fonction zêta et distributions. In Séminaire Bourbaki, Vol. 9, Exp. No. 312, Soc. Math. France, Paris 1995, 523--531. Zbl 0226.12008 MR 1610983 http://archive.numdam.org/article/SB_1964-1966_9_523_0.pdf

Received February 22, 2010; revised March 29, 2011

T. Crisp, Institut for Matematiske Fag, University of Copenhagen, Universitetsparken 5, 2100 København Ø, Denmark

E-mail: crisp@math.ku.dk 